\title{
Particles and Scaling for Lattice Fields and Ising Models
}

\author{
James Glimm* \\ Rockefeller University, New York, N. Y. 10021, USA \\ Arthur Jaffe ${ }^{\star \star}$ \\ Harvard University, Cambridge, Mass. 02138, USA
}

\begin{abstract}
The conjectured inequality $\Gamma^{(6)} \leqq 0$ leads to the existence of $\varphi_{d}^{4}$ fields and the scaling (continuum) limit for $d$-dimensional Ising models. Assuming $\Gamma^{(6)} \leqq 0$ and Lorentz covariance of this construction, we show that for $d \geqq 6$ these $\varphi_{d}^{4}$ fields are free fields unless the field strength renormalization $Z^{-1}$ diverges. Let $\lambda$ be the bare charge and $\varepsilon$ the lattice spacing. Under the same assumptions $\left(\Gamma^{(6)} \leqq 0\right.$, Lorentz covariance and $d \geqq 6$ ) we show that if $\lambda \varepsilon^{4-d}$ is bounded as $\varepsilon \rightarrow 0$, then $Z^{-1}$ is bounded and the limit field is free.
\end{abstract}

\section{Introduction}

Even $\varphi^{4}$ fields in the single phase region describe particles which interact through repulsive forces. The evidence in support of this statement includes the absence of even bound states $[24,3,34]$ the canonical lower bound on critical exponents [16], the arguments of [19] concerning absence of three-particle bound states and $C D D$ zeros (which depend on the conjectured inequality $\Gamma^{(6)} \leqq 0$ ) and the scaling and numerical arguments of $[22,29]$ in favor of this $\Gamma^{(6)}$ inequality for $d \leqq 3$.

The purpose of this note is to extend some of these ideas to lattice $\varphi^{4}$ field theories and Ising models. There are two reasons for studying lattice (as opposed to continuum) field theories. The first reason is that all dimensions, including $d \geqq 4$, are possible, and that the results obtained may be related to the existence $[17,16,1,30,21]$ and triviality/nontriviality problems in these dimensions, see also $[32,33]$. The second reason is that the lattice $\varphi^{4}$ field theory is an intermediate link between the continuum $\varphi^{4}$ field theory and the Ising model, and may serve to clarify the relation between Ising model and field theory critical behavior, see $[20-22,26-27,31]$. Beyond these two reasons, we note that lattice field theories are at the outset easier because of the absence of ultraviolet problems but ultimately more difficult because of the absence of the Euclidean rotation symmetry.

* Supported in part by the National Science Foundation under Grant MPS 74-13252

$\star \star$ Supported in part by the National Science Foundation under Grant MPS 75-21212 


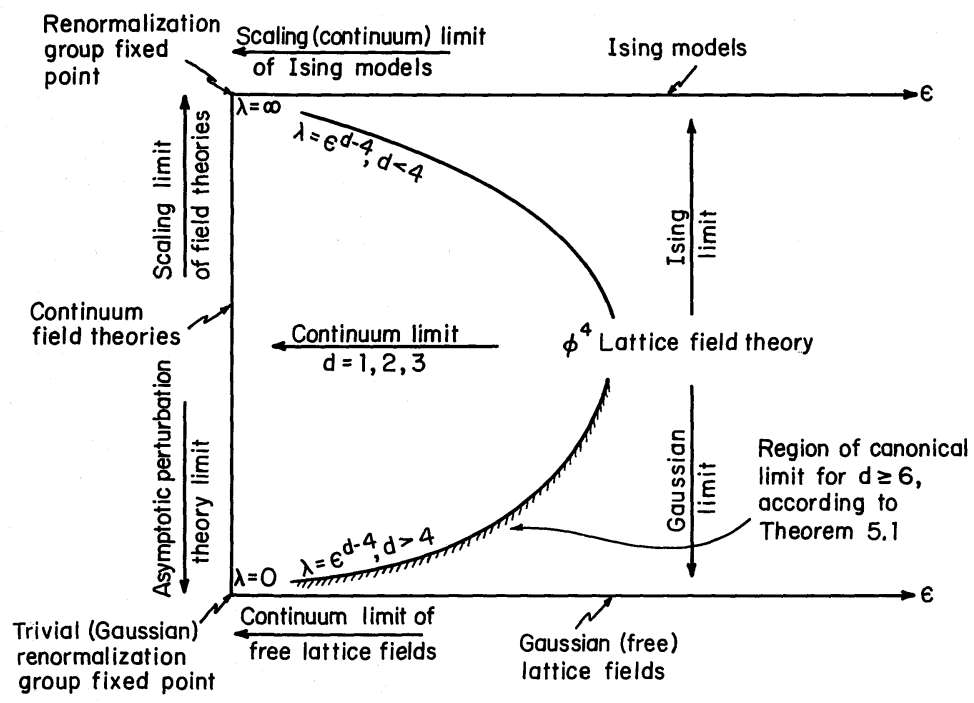

Fig. 1. The $\lambda \varphi^{4}+\sigma \varphi^{2}$ parameter plane. $\varepsilon=$ lattice spacing; $\sigma=\sigma(\lambda, \varepsilon, m)$ chosen to give mass $m$ in single phase region; $m$ held fixed (e.g. $m=1$ ). The $\lambda$ axis represents (continuum) field theory. The $\varepsilon$ axis at $\lambda=\infty$ represents Ising models [30]. The $\varepsilon$ axis at $\lambda=0$ represents Gaussian lattice fields. The curve $\lambda=\varepsilon^{d-4}$ represents constant dimensionless bare charge. Charge renormalization for $d \geqq 4$ may lead to a reparametrization of the $\lambda$ axis

As a compensation for the loss of Euclidean invariance and of the resulting Lehmann spectral formula, we use the momentum space canonical upper bound [9] on the two point function, as well as the $\Gamma^{(6)} \leqq 0$ inequality as used in [19].

Our first main result assumes only $\Gamma^{(6)} \leqq 0$. The conclusion is the existence of a continuum $\varphi_{d}^{4}$ field theory with an arbitrary choice of charge renormalization. Included is the existence of the scaling (continuum) limit for Ising models. The second main result assumes in addition Lorentz invariance of the continuum theory, as well as the (finite) charge renormalization, $\lambda \leqq \varepsilon^{d-4}, \varepsilon$ being the lattice spacing. Note that $\varepsilon^{d-4} \rightarrow 0$ in the continuum limit $\varepsilon \rightarrow 0$, for $d \geqq 5$. In this case, the $\varepsilon=0$ theory is canonical for $d \geqq 6(\S 5)$.

The limits considered here are summarized in Figure 1 above. Coincidence of the two fixed points seems to correspond to the picture in which nontrivial $\varphi^{4}$ field theories cannot be constructed as a limit of lattice cutoff approximations. Similarly, it would seem that the Ising model would be governed by the trivial fixed point only in the case for which a nontrivial fixed point does not occur in Figure 1.

A renormalizability curve $\lambda=0\left(\varepsilon^{d-4}\right)$ of constant dimensionless bare charge is indicated on this figure. A limit constructed along any such curve is renormalizable. Those limits constructed below this family of curves are superrenormalizable (e.g. the continuum limit with constant $\lambda, d=1,2,3)$. Theories constructed above these curves are nonrenormalizable (i.e. $\lambda \varepsilon^{4-d} \rightarrow \infty$ as $\varepsilon \rightarrow 0$ ). The charge $\lambda$ has dimension (length) ${ }^{d-4}$, so that $\lambda \varepsilon^{4-d}$ is a dimensionless bare charge. 


\section{The Wave Function Renormalization and Proper Self Energy for Noncritical Lattice Theories}

In this chapter, we consider a $P(\varphi)$ lattice field or Ising model. We suppose that the infinite volume limit is defined and is invariant under lattice translations, rotations and reflections. We also assume $F K G$ inequalities [6], a positive mass $m>0$ (i.e. an exponential decay rate for correlations in lattice directions) and a single phase. These restrictions should be viewed as restrictions on the boundary conditions and/or coupling constants. In particular they are satisfied for $\varphi^{4}$ interactions and the Ising model approaching the critical point from a single phase direction. Let

$$
G^{(2)}(x)=\langle\varphi(x) \varphi(0)\rangle-\langle\varphi(x)\rangle\langle\varphi(0)\rangle \geqq 0
$$

be the truncated two point function. The Fourier transform

$$
G^{(2)}(p)=\sum e^{-i p x} G^{(2)}(x)
$$

is nonnegative because $G^{(2)}(x)$ is positive definite. Using invariance of $G^{(2)}(x)$ under reflections and rotations, $G^{(2)}(p)$ is a symmetric function of the variable $\left\{\cos p_{v}\right\}_{v=1}^{d}$. Using the selfadjoint transfer matrix, one shows that

$$
G^{(2)}\left(x_{1}, \ldots, x_{d}\right) \leqq G^{(2)}\left(0, \ldots 0, x_{v}, \ldots 0\right)
$$

for any $v$ [30]. In particular $G^{(2)}$ has an exponential decay rate in all directions and $G^{(2)}$ is analytic for $p$ in a tube $\tau$. By Hartog's theorem, $\tau$ is convex and also using the $p_{v} \rightarrow-p_{v}$ symmetry, we see that

$$
\left\{p: \sum_{v=1}^{d}\left|\operatorname{Im} p_{v}\right| \leqq m\right\} \subset \tau \subset\left\{p: \underset{v}{\left.\sup \left|\operatorname{Im} p_{v}\right| \leqq m\right\}}\right. \text {. }
$$

The use of Hartog's theorem can be avoided by a Schwarz inequality. For $p_{1}, p_{2} \in \tau$,

$$
\left|\sum G^{(2)}(x) e^{-i\left(p_{1}+p_{2}\right) x / 2}\right| \leqq\left(\sum G^{(2)}(x) e^{\operatorname{Im} p_{1} x}\right)^{\frac{1}{2}}\left(\sum G^{(2)}(x) e^{\operatorname{Im} p_{2} x}\right)^{\frac{1}{2}} .
$$

Thus not only is $\tau$ convex, but its border coincides with the singularities of $G^{(2)}(p)$, for $\operatorname{Re} p=0$. We have proved the following:

Proposition 2.1. For a lattice field or Ising model as above, $G^{(2)}$ is analytic in a convex tube $\tau$, satisfying (2.4).

We distinguish a single lattice direction $x$, and write $\boldsymbol{x}=\left\{x_{2}, \ldots, x_{d}\right\}, \boldsymbol{p}=$ $\left\{p_{2}, \ldots, p_{d}\right\}$. The existence of a self adjoint transfer matrix means that $G^{(2)}$ has an analytic continuation for $p_{1}$ in the half plane $p_{1}^{2} \neq$ neg. real, for $\boldsymbol{p}$ real.

Proposition 2.2. Consider a lattice field or Ising model as above with p real. Then $G^{(2)}(p)$ is Herglotz as a function of the variable $1-\cos p_{1}$. For some positive measure d@( $a, \boldsymbol{p})$ depending on $\boldsymbol{p}$,

$$
G^{(2)}(p)=\int_{-1+\cosh m}^{\infty} \frac{d \varrho(a, p)}{1-\cos p_{1}+a},
$$


and

$0<\int \frac{d \varrho}{a}<\infty$.

Proof. An explicit calculation shows that the Fourier transform of $e^{-\mu|n|}$ is

$\Sigma_{n} e^{-\mu|n|+i p n}=\sinh \mu /(\cosh \mu-\cos p)$.

Substitution of this formula into the spectral representation for the transfer matrix establishes (2.5) after the change of variable $a=-1+\cosh \mu$, while the upper bound in (2.6) follows from

$$
\sum_{x} G^{(2)}(x) e^{-i \boldsymbol{p} \boldsymbol{x}}=G^{(2)}(0, \boldsymbol{p})=\int \frac{d \varrho(a, \boldsymbol{p})}{a} \leqq \sum_{x} G^{(2)}(x)<\infty .
$$

From (2.5) we see that $G^{(2)}(p)$ is monotone decreasing in each argument $p_{v}$ for $0 \leqq p_{v} \leqq \pi$. We show that $G^{(2)}(\pi, \ldots, \pi) \neq 0$ and then it follows from (2.5) and monotonicity that $d \varrho(a, p)$ is nonzero for any real $p$. If $G^{(2)}(\pi, \ldots, \pi)=0$, then $d \varrho(a, \pi)=0$ by $(2.5)$, and so $G^{(2)}\left(p_{1}, \pi, \ldots, \pi\right)=0$ for all $p_{1}$. Interchanging first and second coordinates, $G^{(2)}\left(\pi, p_{2}, \pi, \ldots, \pi\right)=0$, and so $G^{(2)}\left(p_{1}, p_{2}, \pi, \ldots, \pi\right)=0$ for arbitrary $p_{1}$ and $p_{2}$. Continuing in this fashion, we conclude that $G^{(2)}$ and $G^{(2)}$ both vanish, which is a contradiction.

Definition. $\Gamma^{(2)}(x)$ is the convolution inverse to $-G^{(2)}$, so that

$$
\Gamma^{(2)}(p)=-1 / G^{(2)}(p) .
$$

The next result is elementary, cf. [19].

Proposition 2.3. Consider a lattice field theory or Ising model as above. $\Gamma^{(2) \sim}$ is analytic in a convex tube $\tau_{\Gamma}$ satisfying

$$
\left\{p: \sum\left|\operatorname{Im} p_{v}\right| \leqq m\right\} \subset \tau_{\Gamma} .
$$

For $\boldsymbol{p}$ real, $\Gamma^{(2)}$ is Herglotz as a function of $1-\cos p_{1}$, and has a unique representation

$$
\begin{aligned}
\Gamma^{(2)}(p)= & -\alpha(\boldsymbol{p})\left(1-\cos p_{1}\right) \\
& +\beta(\boldsymbol{p})+\int_{-1+\cosh m}^{\infty}\left[\frac{1}{1-\cos p_{1}+a}-\frac{a}{a^{2}+1}\right] d v(a, \boldsymbol{p})
\end{aligned}
$$

with $\alpha \geqq 0, \beta$ real, $d v$ positive and

$$
\int d v /\left(1+a^{2}\right)<\infty .
$$

Remark. For $d \geqq 3$, there is a constant $K$ depending only on $d$ and $G^{(2)}(0)$ such that

$$
\alpha+|\beta|+\int\left(1+a^{2}\right)^{-1} d \nu \leqq K .
$$

Proof. By [9],

$$
G^{(2)}(p) \leqq \text { const. }|p|^{-2} \text {. }
$$


By the Herglotz property, used successively in each coordinate, as in the proof of Proposition 2.2,

$$
G^{(2)}(p) \leqq \text { const. } \prod_{\nu=1}^{d}\left|p_{\nu}\right|^{-2} G^{(2)}(\pi, \ldots, \pi) .
$$

Thus for any $r>0$ and for $d>3$,

$$
\begin{aligned}
G^{(2)}(0)= & (2 \pi)^{-d} \int G^{(2)}(p) d p \\
& \leqq \text { const. }\left(\int_{\substack{\operatorname{Inf}\left|p_{v}\right| \leqq r\\
}}|p|^{-2} d p+r^{-2 d} G^{(2)}(\pi, \ldots, \pi)\right) \\
& \leqq \text { const. }\left(r+r^{-2 d} G^{(2)}(\pi, \ldots, \pi)\right) .
\end{aligned}
$$

Here the optimal choice of $r$ is

$$
r=0\left(G^{(2)}\right)(\pi, \ldots, \pi)^{1 /(2 d+1)}
$$

and so

$$
G^{(2)}(0)^{2 d+1} \leqq \text { const. } G^{(2)} \mathcal{\sim}(\pi, \ldots, \pi) \leqq \text { const. } G^{(2)}(p) .
$$

A uniform upper bound on (2.9) follows. For $d=3$, the exponents must be changed slightly to dominate $r \ln r$ in place of $r$, but a bound on (2.9) in still valid. Linear combinations of $\cos p_{1}=0,-1$ show that $\beta$ and $\alpha+\int\left(a^{2}+1\right)^{-1} d v(a)$ are each bounded. Since $\alpha$ and $d v$ are each nonnegative, the individual terms $\alpha$ and $\int\left(a^{2}+1\right)^{-1} d v$ are also bounded, proving $(2.10)$.

We now write $x=\left(x^{\prime}, x^{\prime \prime}\right), p=\left(p^{\prime}, p^{\prime \prime}\right)$ for some decomposition

$$
x^{\prime}=\left(x_{1}, \ldots, x_{j}\right), \quad x^{\prime \prime}=\left(x_{j+1}, \ldots, x_{d}\right)
$$

of the coordinates, and consider the partial Fourier transform

$$
\Gamma^{(2)}\left(x^{\prime}, p^{\prime \prime}\right) \equiv \sum_{x^{\prime \prime}} e^{-i p^{\prime \prime} x^{\prime \prime}} \Gamma^{(2)}\left(x^{\prime}, x^{\prime \prime}\right) \text {. }
$$

Proposition 2.4. Consider a lattice field or Ising model as above. For $x_{1} \neq 0$, $x_{2}=\ldots=x_{j}=0, p^{\prime \prime}$ real, $\Gamma^{(2)}\left(x^{\prime}, p^{\prime \prime}\right)$ is positive and has an exponential decay rate $\bar{m} \geqq m$. (Note $j \neq 0$, by assumption.)

Proof. First we consider the case $x^{\prime}=x_{1}$ and $p^{\prime \prime}=\boldsymbol{p}$. The Herglotz representation (2.9) in $x_{1}$-space has three terms. The first is nonnegative for $x_{1} \neq 0$. The second, proportional to $\beta$, vanishes for $x_{1} \neq 0$. The third is positive for all $x_{1}$, so the proof is complete in this case. The general case follows from the case just considered, because integration with respect to $p_{2}, \ldots, p_{j}$ is equivalent to evaluation at $x_{2}=\ldots=x_{j}=0$.

Definition. If the measure $d \varrho(a, \boldsymbol{p}=0)$ has a $\delta$-function at $a=-1+\cosh m$, then $Z>0$ is the strength of this $\delta$-function. Otherwise by definition $Z=0$.

Proposition 2.5. For a pure phase lattice $P(\varphi)$ field theory or Ising model with $m>0$ and $Z>0$,

$$
\begin{aligned}
Z^{-1} & =-\left.\left(\partial / \partial\left(1-\cos p_{1}\right)\right) \Gamma^{(2)}\left(p_{1}, \boldsymbol{p}\right)\right|_{\cos p=\cosh m, \boldsymbol{p}=0} \\
& =\alpha(0)+\int_{0}^{\infty}(a-\cosh m+1)^{-2} d v(a, 0)
\end{aligned}
$$


Proof. We write

$$
G^{(2)}\left(p_{1}, 0\right)=Z\left(\cosh m-\cos p_{1}\right)^{-1}+\int \frac{d \hat{\varrho}}{1-\cos p_{1}+a}
$$

where $\int_{-1+\cosh m}^{-1+\cosh m+\varepsilon} d \hat{\varrho} \rightarrow 0$ as $\varepsilon \rightarrow 0$. Thus as $\cos p_{1} \rightarrow \cosh m$,

$$
Z\left(\cosh m-\cos p_{1}\right)^{-1} \leqq G^{2 \sim}\left(p_{1}, 0\right) \leqq(Z+\varepsilon)\left(\cosh m-\cos p_{1}\right)^{-1}
$$

and the derivative of $\Gamma^{(2)}$ can be evaluated as $Z^{-1}$ from this inequality.

Remark. We have not assumed an upper mass gap, in contrast to [19].

Definition. $C_{L, m_{0}}$ is the free (Gaussian) lattice covariance,

$$
C_{L, m_{0}}=\left[-1+\cosh m_{0}+\sum_{v=1}^{d}\left(1-\cos p_{v}\right)\right]^{-1}
$$

and

$$
\Pi(p)=\Gamma^{(2)} \mathcal{\gamma}(p)+C_{L, m_{0}}^{-1} .
$$

Proposition 2.6. Consider a lattice field theory with the hypotheses of $\$ 2$. Let $P_{0}, P_{1}$ be projections onto the vacuum $\Omega(\equiv 1)$ and the span of $\left(1-P_{0}\right) \varphi \Omega$ respectively. Then

$$
\Pi(x)=-\left\langle P^{\prime \prime}\right\rangle \delta_{0}(x)+\left\langle P^{\prime}(x),\left(1-P_{0}-P_{1}\right) P^{\prime}(0)\right\rangle
$$

if the Wick ordering covariance for $P$ is $C_{L, m_{0}}$.

Proof. As in [19]. The Ising model is excluded because the $\delta$-functions in the single spin measure prevent integration by parts.

Proposition 2.7. Consider a lattice field theory as above.

$$
\left.\Gamma^{(2)}\left(x_{1}, \ldots, x_{j}, p_{j+1}, \ldots, p_{d}\right)\right|_{x_{1}=0=\ldots=x_{j}} \geqq 1-\cosh m_{0}-\left\langle P^{\prime \prime}\right\rangle-2 d
$$

Proof. We use the integration by parts representation (2.13-14), and omit

$$
\sum_{x^{\prime \prime}} e^{-i p^{\prime \prime} x^{\prime \prime}}\left\langle P^{\prime}\left(x^{\prime}=0, x^{\prime \prime}\right)\left(1-P_{0}-P_{1}\right) P^{\prime}(0)\right\rangle .
$$

Here $x^{\prime}=x_{1}, \ldots, x_{j}$ and $x^{\prime \prime}=x_{j+1}, \ldots, x_{d}$. The omitted quantity is positive because

$$
x^{\prime \prime} \mapsto\left\langle P^{\prime}\left(x^{\prime}=0, x^{\prime \prime}\right)\left(1-P_{0}-P_{1}\right) P^{\prime}(0)\right\rangle
$$

is positive definite and hence the Fourier transform of a positive measure.

\section{Three Particle Decay for $\mathscr{P}(\varphi)=\varphi^{4}$}

In this section we consider $\lambda \varphi^{4}+\sigma \varphi^{2}$ lattice field theories with $\sigma>\sigma_{c}$. Then all hypotheses of $\S 2$ are satisfied. Ising models are excluded because we integrate by parts. 
Proposition 3.1. Assume $\Gamma^{(6)}(x x x y y y) \leqq 0$ for $x-y$ large. Then the CDD radius $\bar{m}$ satisfies $\bar{m} \geqq 3 m$. The analyticity tube $\tau_{\Gamma}$ for $\Gamma^{(6)}$ satisfies

$$
\left\{p: \Sigma\left|\operatorname{Im} p_{v}\right| \leqq 3 m\right\} \subset \tau_{\Gamma}
$$

Proof. As in [19]. See also Proposition 3.2 below.

This result leads to the existence of $\varphi_{d}^{4}$ field theories and the scaling limit for Ising models, see $\S 4$. In order to establish the triviality of some of these field theories, we need to assume canonical upper bounds for the two point decay rate (as would follow from the Lehmann spectral formula in the continuum case). Explicitly, we assume

$$
0 \leqq G^{(2)}(x) \leqq\left\{\begin{array}{l}
K_{1}|x|^{-d+2} e^{-m|x|},|x| \leqq m^{-1} \\
K_{1} m^{d-2} e^{-m|x|},|x| \geqq m^{-1}
\end{array}\right.
$$

This bound is always satisfied for fix ed $\sigma, \lambda$, but eventually we will consider the postulate that $K_{1}$ is independent of $\sigma$ and/or $\lambda$, which is not known.

Proposition 3.2. Assume (3.1) and $\Gamma^{(6)}(x x x y y y) \leqq 0$ for $a \lambda \varphi^{4}+\sigma \varphi^{2}$ lattice field theory with $\sigma>\sigma_{c}$. Then for

$$
K=\left(K_{1}^{3}+K_{1}^{4}\right) K_{2}\left(\left|\cosh m_{0}+\left\langle P^{\prime \prime}\right\rangle\right|+1\right)
$$

where $K_{2}$ is a universal constant and $K_{1}$ is defined by (3.1),

$$
\Pi\left(x_{1}, \boldsymbol{p}=0\right) \leqq\left\{\begin{array}{l}
\lambda^{2} K\left|x_{1}\right|^{-2 d+7} e^{-3 m\left|x_{1}\right|} \text { for }\left|x_{1}\right| \leqq m^{-1} \\
\lambda^{2} K m^{2 d-7} e^{-3 m\left|x_{1}\right|} \text { for }\left|x_{1}\right| \geqq m^{-1}
\end{array}\right.
$$

Proof. As in [19], we expand $\Pi$ in terms of connected and one particle irreducible subdiagrams. Negative terms, including $\Gamma^{(6)}$ can be omitted, yielding the bound

$$
\Pi\left(x_{1}, \boldsymbol{p}=0\right) \leqq \sum_{\boldsymbol{x}} \lambda^{2}\left\{6 G^{(2)}(x)^{3}-36 \sum_{z, z^{\prime}} G^{(4)}(x x 0 z) \Gamma^{(2)}\left(z-z^{\prime}\right) G^{(4)}\left(z^{\prime} 00 x\right)\right\} .
$$

By Lebowitz' inequality, $G^{4} \leqq 0$. Let $p_{j}$ be the momentum variable conjugate to $z_{j}-z_{j}^{\prime}$. By Proposition 2.4, applied with $p^{\prime \prime}=\left(p_{2}, \ldots, p_{d}\right)=0$, the terms with $z_{1}-z_{1}^{\prime} \neq 0$ may be omitted. Having done so, we apply Proposition 2.4 again with $z_{1}-z_{1}^{\prime}=0, p^{\prime \prime}=\left(p_{3}, \ldots, p_{d}\right)=0$; we omit terms with $z_{2} \neq z_{2}^{\prime}$. Continuing in this fashion we have

$$
\Pi\left(x_{1}, \boldsymbol{p}=0\right) \leqq \sum_{x} \lambda^{2}\left\{6 G^{(2)}(x)^{3}+36\left(2 d+1+\left\langle P^{\prime \prime}\right\rangle\right) \sum_{x} G^{(4)}(x x 0 z) G^{(4)}(z 00 x)\right\},
$$

where Proposition 2.7 provides the lower bound on $\Gamma^{(2)}(0)$. Lebowitz' inequality bounds the $G^{(4)}$ factors above, so that

$$
\begin{aligned}
\Pi\left(x_{1}, \boldsymbol{p}=0\right) & \leqq \sum_{\boldsymbol{x}} \lambda^{2}\left\{6 G^{(2)}(x)^{3}\right. \\
& \left.+144\left(2 d-1+\left\langle P^{\prime \prime}\right\rangle\right) G^{(2)}(x)^{2}\left(G^{(2)} * G^{(2)}\right)(x)\right\} .
\end{aligned}
$$

Substitution of (3.1) yields (3.2). 


\section{Existence of Continuum $\varphi_{d}^{4}$ Fields and the Ising Scaling Limit}

The original existence, regularity and Lorentz covariance theorems for $\varphi_{2}^{4}$ fields were given in $[10-12,15,2]$. A survey of this work, including the contributions of Nelson, Segal and others may be found in $[13,14]$. The remaining two Wigthman axioms - uniqueness of the vacuum and its invariance under Lorentz rotations - were verified in [23] for weak coupling, and in [8] for arbitrary coupling. The completion of the $\varphi_{3}^{4}$ existence program has recently been achieved, see $[4,28]$ for weak coupling and $[5,7]$ for arbitrary coupling. The original nonuniqueness proof for relativistic fields was given in [25].

In this section we consider the continuum limit of lattice $\varphi_{d}^{4}$ fields.

Theorem 4.1. Assume $\Gamma^{(6)}(x x x y y y) \leqq 0$ for all $\sigma>\sigma_{c}(\lambda)$ and all $\lambda$ for $\lambda \varphi^{4}+\sigma \varphi^{2}$ lattice fields. Choose a mass $m>0$, and for each choice of lattice spacing $\varepsilon>0$, choose a bare charge $\lambda(\varepsilon)$. Then the limit $\varepsilon \rightarrow 0$ defines (through convergent subsequences) a continuum field theory limit of the $\lambda(\varepsilon) \varphi^{2}+\sigma \varphi^{2} \varepsilon$-lattice theories. This limit has mass $m>0$ and satisfies the Wightman-Osterwalder-Schrader axioms with the possible exception of Lorentz invariance.

Proof. By $[1,30]$, there is a $\sigma=\sigma(\varepsilon)>\sigma_{c}(\lambda(\varepsilon))$ which gives mass $m>0$ to the $\varepsilon$-lattice field theory. By [17], existence of the limit (through subsequences) follows from a uniform bound on the two point function. As in [19], the bound on the CDD radius gives the required bound on the wave function renormalized two point function. Because of the wave function renormalization, the limit theory has a mass $m$. This completes the proof.

A portion of this proof extends to Ising models.

Proposition 4.2. Assume $\bar{m}$ (the $C D D$ radius) $\geqq(1+\delta) m, \delta>0$, for $T>T_{c}$ in the $d$-dimensional Ising model. Then the scaling limit exists and defines a field theory in the sense specified in Theorem 4.1.

Theorem 4.3. Assume $\Gamma^{(6)}(x x x y y y) \leqq 0$ for $\lambda \varphi^{4}+\sigma \varphi^{2}$ fields with mass $m$ fixed, positive and $\lambda$ sufficiently large $\left(\sigma>\sigma_{c}\right)$. Then the Ising model scale limit exists.

Proof. By [30], the Schwinger functions of the lattice field theory converge, as $\lambda \rightarrow \infty$ to the Schwinger functions of the Ising model. Thus $G^{(2)}(p)$ and $\Gamma^{(2)}(p)$ converge for real $p$. In fact from (2.3), (2.7) and $m>0$ we have a uniform bound on $\int d \varrho(a, p) / a$ which implies pointwise convergence of $G^{(2)}(p)$ for real $p$. As in the proof of Proposition 2.2, $G^{(2)}(p)$ is bounded away from zero, so $\Gamma^{(2)} \mathcal{r}(p)$ converges pointwise and is bounded also. However the remark following Proposition 2.3 yields bounds on $\alpha,|\beta|$ and $\int d v /\left(1+a^{2}\right)$, which by Propositions 2.3 and 3.1 imply that $\Gamma^{(2)}\left(p_{1}, 0\right)$ is analytic up to $\left|\operatorname{Im} p_{1}\right|<3 m$, and bounded uniformly in $\lambda$. Hence the same is true for the $\Gamma^{(2)}$ of the Ising model. Thus $\bar{m} \geqq 3 m$. This statement is uniform in $m$, and existence of the scaling limit follows from Proposition 4.2.

\section{Critical $\varphi^{4}$ Lattice Theories, $d \geqq 6$}

Theorem 5.1. Consider a $\lambda \varphi^{4}+\sigma \varphi^{2}$ lattice field theory with $\sigma>\sigma_{c}(\lambda)$ and $\Gamma^{6}(x x x y y y) \leqq 0$. Then $Z \neq 0$. For $d \geqq 6$, the bound on $Z^{-1}$ is independent of $\sigma>\sigma_{c}(\lambda)$ and $\lambda$ for $\lambda$ and $m$ bounded. 
Proof. $Z>0$ because $\Gamma^{(6)} \leqq 0$ implies an upper mass gap. To establish the uniform bound on $Z^{-1}$ for $d \geqq 6$, we use (2.12). By the remark following Proposition 2.3, it is sufficient to estimate the small $a$ behavior of the $d v$ integral, which we do using (2.11), [9] and (3.4). From (2,12),

$$
\begin{aligned}
& Z^{-1}=\text { const. }+\int_{a=1}^{\infty} \frac{d v}{-\cosh m} \frac{d v}{(a+1-\cosh m)^{2}} \leqq \text { const. }+ \text { const. } \int a^{-2} d v \\
& \leqq \text { const. }\left(1-\left.\left(\frac{\partial}{\partial p_{1}}\right)^{2} \Gamma^{-}(p)\right|_{p=0}\right) \text {. }
\end{aligned}
$$

(Note in general $\left.\left.\partial / \partial p_{1}^{2}\right) \neq\left(\partial / \partial p_{1}\right)^{2}\right)$. By $(2.13)-(2.14)$,

$$
Z^{-1} \leqq \text { const. }\left(1-\left.\left(\frac{\partial}{\partial p_{1}}\right)^{2} \Pi^{\sim}(p)\right|_{p=0}\right)
$$

and by (3.4)

$$
\begin{aligned}
Z^{-1} & \leqq \text { const. }\left(1+\sum_{x} x_{1}^{2} \Pi(x)\right) \\
& \leqq \text { const. }\left[1+\sum_{x} x_{1}^{2}\left(G^{(2)}(x)^{3}+G^{(2)}(x)^{2} G^{(2)} * G^{(2)}(x)\right)\right] \\
& \leqq \text { const. }\left.\left[1-\left(\frac{\partial}{\partial p_{1}}\right)^{2}\left(G^{(2) \sim} * G^{(2)} * G^{(2)}+G^{(2)} * G^{(2)} *\left(G^{(2)}\right)^{2}\right)\right]\right|_{p=0} .
\end{aligned}
$$

The point to these manipulations is to replace a derivative of $\Gamma$ by a derivative of the upper bound on $\Gamma$. By the Herglotz property, we are again able to transfer derivatives to the upper bound (2.11) as follows. For simplicity, we consider only the final (and worst) term above. It is

$$
\begin{aligned}
& -\left(\frac{\partial}{\partial p_{1}}\right)^{2} \int\left(\cos (p-k)^{2}+a\right)^{-1}\left(\cos (k-l)^{2}+b\right)^{-1}\left(\cos (l-q)^{2}+c\right)^{-1}\left(\cos (l-q)^{2}+e\right)^{-1} \\
& \times\left. d \varrho(a) d \varrho(b) d \varrho(c) d \varrho(e) d k d l d q\right|_{p=0} .
\end{aligned}
$$

Without loss of generality, we restrict all $d \varrho(a) \ldots d \varrho(e)$ integrations to the interval $[0,1]$. By $(2.11)$, it follows that

$$
\int_{0}^{1} d \varrho(a, 0) \leqq \text { const. }
$$

and so we may replace the $d \varrho$ integrations by $\sup _{a, b, c, e}$. This supremum is evaluated as $0=a=b=c=e$. Thus we have accomplished our goal. The derivative is placed on the upper bound, in this case the zero mass free lattice field. It is convenient to return to $x$-space, where we find

$$
Z^{-1} \leqq \text { const. }\left[1+\sum_{x} x_{1}^{2}\left(x^{-3 d+6}+\sum_{y}(x-y)^{-d+2} y^{-d+2}\right) x^{-2 d+4}\right]
$$

The theorem follows from this bound.

Theorem 5.2. Under the hypothesis of Theorem 5.1, and the construction of Theorem 4.1, the two point function is free $(\Pi=0)$ at momentum $\boldsymbol{p}=0$, in the limit $\varepsilon \rightarrow 0$. 
Remark. If $\lambda=\lambda(\varepsilon)$ is the bare charge ( $\varepsilon=$ lattice spacing) then $\lambda(\varepsilon) \leqq \varepsilon^{d-4}$ in the above case.

Corollary 5.3. Suppose in addition that the limit $\varepsilon \rightarrow 0$ above is Euclidean invariant. Then it is a free scalar field of mass $m$.

Proof. The two point function is free by the theorem, and the theory as a whole is free by $[35, \mathrm{p} .163]$.

Proof of Theorem 5.2. Let $\Gamma_{\lambda, m, \varepsilon}^{\sim}(p)$ be the inverse propagator for the theory with bare charge $\lambda$, mass $m$ and lattice spacing $\varepsilon$. By scaling,

$$
\Gamma_{s^{d-4} \lambda, s^{-1} m, s \varepsilon}(p)=s^{-2} \Gamma_{\lambda, m, \varepsilon}(s p) .
$$

Here we choose $\varepsilon=1, s \rightarrow 0$, and

$$
\begin{aligned}
& s^{-1} m=\text { const., i.e. } \quad m=\text { const. } s \rightarrow 0 \\
& s^{d-4} \lambda=\lambda(s) \leqq 0\left(s^{d-4}\right)
\end{aligned}
$$

so that the $\lambda$ on the right side of (5.2) is bounded, but possibly $s$-dependent as $s \rightarrow 0$. Now $\Gamma_{\lambda, m, \varepsilon}\left(p_{1}, 0\right)$ has a zero at $\cos p=\cosh m$ and as we have just seen, bounded first and second derivatives with respect to $p_{1}$ evaluated at $\boldsymbol{p}=0, p_{1}=0(m)$. Furthermore the first derivative vanishes at $p_{1}=0$ because of the $p_{1} \rightarrow-p_{1}$ symmetry. The same method bounds one more $p_{1}$ derivative, and

$$
\left.s^{-2} \Gamma_{\lambda, m, \varepsilon}(s p)\right|_{p=0}=s^{-2}\left(Z^{-1}\left(\cosh m-\cos s p_{1}\right)+0\left(s^{3} p_{1}^{3}\right)\right) \rightarrow Z^{-1}\left(p_{1}^{2}+\text { const. }\right)
$$

as $s \rightarrow 0$. This completes the proof.

Remark. The vanishing of $\Gamma$ on the mass shell gives a cancellation which formally allows $d \geqq 5$ in place of $d \geqq 6$ above.

\section{Upper Bounds on Critical Exponents}

We give upper bounds on certain critical exponents in a single phase $\varphi^{4}+\sigma \varphi^{2}$ (lattice) quantum field model. In particular we study the anomalous dimension $\eta$, the exponent $\zeta$ for the field strength renormalization constant $Z$ and the exponent $v$ for the physical mass. We choose $\sigma>\sigma_{c}$ (the single phase region) and consider the limit $\sigma \searrow \sigma_{c}$.

In this section we assume $\Gamma^{(6)} \leqq 0$, as well as the $x$-space upper bound

$$
G^{(2)}(x, \sigma) \leqq K\left(\min \left\{|x|, m^{-1}\right\}\right)^{-(d-2+\eta)} e^{-m|x|}
$$

with $K$ uniform in the allowed $x, \sigma$. We expect (6.1) to hold for lattice field theories. We then show $\eta=0=\zeta$ for $d \geqq 5$, complementing the results of $\S 5$.

Proposition 6.1. For a pure phase $\lambda \varphi^{4}+\sigma \varphi^{2}$ (lattice) field theory with $m>0, Z>0$ and satisfying (6.1), we have

$$
0 \leqq \eta \leqq \zeta / v \text {. }
$$

If in addition $\Gamma^{(6)} \leqq 0$, then

$$
\zeta / v \leqq 2
$$


Here $\zeta$ is the exponent for the field strength renormalization constant and $v$ is the exponent for the mass. Thus $Z \sim m^{\zeta / v}$ as $m \rightarrow 0$.

Proof. Using (2.12a) evaluated at $p_{1}=0$ gives

$$
m^{-2} \leqq \text { const. } Z^{-1} G^{2 \sim}(0, \sigma) \text {. }
$$

We bound $G^{2 \sim}(0, \sigma)$ by integrating (6.1) to obtain

$$
m^{-2} \leqq \text { const. } Z^{-1} m^{\eta-2} \text {. }
$$

Thus

$m^{-\eta} Z \leqq$ const. ,

or

$$
-\eta+\zeta / v \geqq 0 .
$$

Since the inequality $0 \leqq \eta$ is known we have established (6.2).

Assuming $\Gamma^{(6)} \leqq 0$, we may use (5.1). Then

$$
m^{-\zeta / v} \sim Z^{-1} \leqq \text { const. }\left(1+\sum x_{1}^{2} \Pi(x)\right) .
$$

We show $\Sigma \Pi(x) \leqq$ const., from which we infer

$$
1+\sum x_{1}^{2} \Pi(x) \leqq 0\left(m^{-2}\right),
$$

proving $\zeta / v \leqq 2$. The summability of $\Pi(x)$ follows from

$$
\Sigma \Pi(x)=\Pi^{r}(0)=0(1)+\Gamma^{\sim}(0) .
$$

Consider $\sigma$ in the interval $\left(\sigma_{c}, \sigma_{1}\right)$, for $\sigma_{1}>\sigma_{c}$. Since $\Gamma^{(}(0)=-G^{2 \sim}(0)^{-1} \leqq 0$, we need only bound $\Gamma^{2}(0)$ from below. However $G^{2}(x, \sigma)$ is decreasing in $\sigma$ (a consequence of the second Griffiths inequality). Thus for $\sigma>\sigma_{c}$

$$
\sum_{x} G^{2}(x, \sigma)=-\Gamma^{\sim}(0, \sigma)^{-1}
$$

is decreasing in $\sigma$ and $\Gamma^{\sim}(0, \sigma)$ is also decreasing in $\sigma$. Thus $\Gamma^{\sim}\left(0, \sigma_{c}\right) \geqq \Gamma^{\sim}\left(0, \sigma_{1}\right)$.

Theorem 6.2. Assume $\Gamma^{(6)} \leqq 0$ and (6.1) in a $\lambda \varphi^{4}+\sigma \varphi^{2}$ lattice theory. Then

$$
\eta=\zeta / \nu=0 \text { for } d \geqq 5
$$

and for $d \leqq 4$

$$
0 \leqq \eta \leqq 2(1-d / 5)=\left\{\begin{array}{rl}
.4 & d=4 \\
.8 & d=3 . \\
1.2 & d=2
\end{array}\right.
$$

Proof. We use the bound (5.1) for

$$
\begin{aligned}
m^{-\zeta / v} \sim Z^{-1} & \leqq \text { const. }\left(1+\sum_{x} x_{1}^{2} \Pi(x)\right) \\
& \leqq \text { const. }\left(1+\sum_{x} x_{1}^{2}\left[G(x)^{3}+G(x)^{2}(G * G)(x)\right]\right) .
\end{aligned}
$$


Substituting (6.1) we find the $G^{3}$ term in (6.7) sums to

$$
0\left(m^{-8-2 d-3 \eta)}\right)
$$

while the $G^{2}(G * G)$ term sums to

$$
0\left(m^{-(10-2 d-4 \eta)}\right) \text {. }
$$

By Proposition 6.1, 2- $\eta \geqq 0$ and

$$
(10-2 d-4 \eta)-(8-2 d-3 \eta) \geqq 0 \text {. }
$$

Thus the $G^{2}(G * G)$ term in (6.7) dominates and by (6.2), (6.3), (6.7),

$$
0 \leqq \eta \leqq \zeta / v \leqq 10-2 d-4 \eta \text {. }
$$

For $d \geqq 5$,

$$
0 \leqq \eta \leqq \zeta / v \leqq 10-2 d \leqq 0,
$$

proving (6.5). For $d \leqq 4,(6.10)$ gives $5 \eta \leqq 10-2 d$, which is (6.6).

Remark. If the upper bound on $\Pi(x)$ in (3.3) could be improved to show that the $G^{(4)} \Gamma G^{(4)}$ term has the same long distance behavior as the $\left(G^{(2)}\right)^{3}$ term, then the above bounds on $\eta$ are improved to $\eta \leqq \zeta / v \leqq 8-2 d-3 \eta$. In this case $\eta=\zeta=0$ for $d \geqq 4$ (as expected), and for $d \leqq 4, \eta \leqq(2-d / 2)$.

Acknowledgement. We thank T. Spencer and K. Symanzik for helpful conversations.

\section{References}

1. Baker, G.: Self-interacting Boson quantum field theory and the thermodynamic limit in $d$ dimensions. J. Math. Phys. 16, 1324-1346 (1975)

2. Cannon, J., Jaffe, A.: Lorentz covariance of the $\lambda\left(\varphi^{4}\right)_{2}$ quantum field theory. Commun. math. Phys. 17, 261-321 (1970)

3. Feldman, J.: On the absence of bound states for $\lambda \varphi_{2}^{4}$ quantum field models without symmetry breaking. Canad. J. Phys. 52, 1583-1587 (1974)

4. Feldman,J., Osterwalder,K.: The Wightman axioms and the mass gap for weakly coupled $\Phi_{3}^{4}$ quantum field theories. Ann. Phys., to appear

5. Feldman, J., Osterwalder, K.: The construction of $\lambda\left(\varphi^{4}\right)_{3}$ quantum field models, presented at the International Colloquium on Mathematical Methods in Quantum Field Theory, Marseille, June 1975

6. Fortuin,C., Kastelyn,P., Ginibre, J.: Correlation inequalities on some partially ordered sets. Commun. math. Phys. 22, 89-103 (1971)

7. Fröhlich, J.: To appear

8. Fröhlich,J., Simon, B.: Pure states for general $P(\varphi)_{2}$ theories: construction, regularity, and variational equality, preprint, 1976

9. Fröhlich,J., Simon, B., Spencer, T.: Infrared bounds, phase transitions and continuous symmetry breaking. Commun. math. Phys. 50, 79-85 (1976)

10. Glimm, J., Jaffe, A.: A $\lambda \varphi^{4}$ field theory without cutoffs I. Phys. Rev. 176, 1945-1951 (1968)

11. Glimm, J., Jaffe, A.: The $\lambda\left(\varphi^{4}\right)_{2}$ quantum field theory without cutoffs. II. The field operators and the approximate Hamiltonian. Ann. Math. 91, 362-401 (1970)

12. Glimm, J., Jaffe, A.: The $\lambda\left(\varphi^{4}\right)_{2}$ quantum field theory without cutoffs. III. The physical vacuum. Acta Math. 125, 203-267 (1970)

13. Glimm, J., Jaffe, A.: Quantum field models. In: Statistical mechanics (C. DeWitt, R. Stora, eds.), 1970 Les Houches Lectures. New York: Gordon and Breach Science Publishers 1971 
14. Glimm, J., Jaffe, A.: Boson quantum field models. In: Mathematics of contemporary physics. London Mathematical Society (R. Streater ed.). New York-London: Academic Press 1972

15. Glimm,J., Jaffe, A.: The $\lambda \varphi_{2}^{4}$ quantum field theory without cutoffs. IV. Pêrturbations of the Hamiltonian. J. Math. Phys. 13, 1568 -1584 (1972)

16. Glimm, J., Jaffe, A.: The $\varphi_{2}^{4}$ quantum field model in the single phase region: Differentiability of the mass and bounds on critical exponents. Phys. Rev. D 10, 536 - 539 (1974)

17. Glimm, J., Jaffe,A.: A remark on the existence of $\varphi_{4}^{4}$. Phys. Rev. Letters 33, 440 -442 (1974)

18. Glimm, J., Jaffe, A.: On the approach to the critical point. Ann. de 1'Inst. Henri Poincaré 22, $13-26(1975)$

19. Glimm, J., Jaffe, A.: Three particle structure of $\varphi^{4}$ interactions and the infinite scaling limit. Phys. Rev. D 11, 2816-2827 (1975)

20. Glimm, J., Jaffe, A.: Particles and bound states and progress toward unitarity and scaling. Presented at the International Symposium on Mathematical Problems in Theoretical Physics. Kyoto, January 23-29, 1975

21. Glimm, J., Jaffe,A.: Critical problems in quantum fields, presented at the International Colloquium on Mathematical Methods of Quantum Field Theory. Marseille, June 1975

22. Glimm, J., Jaffe, A.: Critical exponents and renormalization in the $\varphi^{4}$ scaling limit, presented at the Conference on Quantum Dynamics: Models and Mathematics. Bielefeld, September 1975

23. Glimm,J., Jaffe, A., Spencer,T.: The Wightman axioms and particle structure in the $P(\varphi)_{2}$ quantum field model. Ann. Math. 100, 585-632 (1974)

24. Glimm,J., Jaffe,A., Spencer,T.: The particle structure of the weakly coupled $P(\varphi)_{2}$ model and other applications of high temperature expansions, Part I: Physics of quantum field models; Part II: The cluster expansion. In: Constructive quantum field theory (G. Velo, A. S. Wightman eds.), 1973 Erice Lectures, Lecture Notes in Physics, Vol. 25. Berlin-Heidelberg-New York: Springer 1973

25. Glimm, J., Jaffe, A., Spencer, T.: Phase transitions for $\varphi_{2}^{4}$ quantum fields. Commun. math. Phys. 45, $293-320(1975)$

26. Isaacson, D.: The critical behavior of the anharmonic oscillator. Commun. math. Phys., to appear

27. McBryan, O., Rosen, J.: Existence of the critical point in $\varphi^{4}$ field theory. Commun. math. Phys., to appear

28. Magnen, J., Sénéor, R.: The infinite volume limit of the $\varphi_{3}^{4}$ model. Ann. l'Inst. H. Poincaré, to appear

29. Marcheson, D.: Private communication

30. Rosen, J.: Mass Renormalization for the $\lambda \varphi^{4}$ Euclidean lattice field theory, preprint, 1975

31. Rosen, J.: The Ising model limit of $\varphi^{4}$ lattice fields, preprint, 1976

32. Schrader, R.: A possible constructive approach to $\varphi_{4}^{4}$. Commun. math. Phys. 49, 131-154 (1976)

33. Schrader, R.: A possible constructive approach to $\varphi_{4}^{4}$ II, preprint, 1976

34. Spencer, T.: The absence of even bound states for $\lambda\left(\varphi^{4}\right)_{2}$. Commun. math. Phys. 39, 77 -79 (1974)

35. Streater, R., Wightman,A. S.: PCT, spin and statistics and all that. New York: Benjamin 1964

\section{Communicated by K. Hepp}

Received May 11, 1976 
